\title{
Author Correction: Investigating the nature of active forces in tissues reveals how contractile cells can form extensile monolayers
}

Lakshmi Balasubramaniam, Amin Doostmohammadi (1), Thuan Beng Saw (1), Gautham Hari Narayana Sankara Narayana, Romain Mueller (1), Tien Dang, Minnah Thomas, Shafali Gupta, Surabhi Sonam, Alpha S. Yap (D), Yusuke Toyama (D),

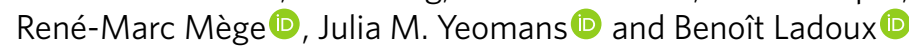

Correction to: Nature Materials https://doi.org/10.1038/s41563-021-00919-2, published online 18 February 2021.

In the version of this Article originally published, the captions for Extended Data Figs. 1, 2 and 3 were in the wrong order and did not correspond to their associated figures. The correct captions are listed below and the Article has been corrected accordingly. In addition, the cell line MCF7 was mistakenly written as 'MCF7A' in seven instances in the main text, Methods and Extended Data Fig. 4 caption, and as 'MCF10A' in one instance in the 'Author contributions' section; these errors have now been corrected.

Extended Data Fig. 1 | MDCK WT behave as an extensile system. a, Kymograph of a short junction $(<10 \mu \mathrm{m})($ top) and long junction $(>15 \mu \mathrm{m})$ (bottom) before and after laser ablation. $\mathbf{b}$, Recoil velocity after laser ablation for short $(<10 \mu \mathrm{m})(\mathrm{n}=9)(\mathrm{N}=4)$, medium $(10-$ $15 \mu \mathrm{m})(\mathrm{n}=13)(\mathrm{N}=4)$ and long junctions $(>15 \mu \mathrm{m})(\mathrm{n}=12)(\mathrm{N}=6) . \mathrm{n}$, is the number of junctions ablated and $\mathrm{N}$ is the number of independent experiments from which these results were obtained. Error bars represent the standard deviation. ANOVA test was performed leading to ${ }^{*} \mathrm{p}<0.05,{ }^{* *} \mathrm{p}<0.01,{ }^{* * *} \mathrm{p}<0.001$ and ${ }^{* * * *} \mathrm{p}<0.0001$. Scale bars, $20 \mu \mathrm{m}$.

Extended Data Fig. 2 | MDCK WT behaves as an extensile system and MDCK E-cadherin KO behave as a contractile system. a,b, Orientation field (left) and velocity vectors (right) around a single comet-shaped (+1/2) defect (a) and trefoil ( $-1 / 2)$ defect obtained from WT (top) and E-cadherin KO (bottom) monolayers. c,d, Trajectory of several comet (+1/2) (left) and trefoil (-1/2) (right) shaped defects obtained from MDCK WT (c) and MDCK E-cadherin KO (d) monolayers. Scale bars: $40 \mu \mathrm{m}$.

Extended Data Fig. 3 Fibroblasts behave as a 2D contractile active nematic. a, Average yy-and xy-components of strain rate map around $+1 / 2$ defect obtained from experiments (left and middle respectively) and corresponding average flow field (right) $(\mathrm{n}=1489$ defects from 2 independent experiments) for NIH3T3 cells. Colour code is positive for stretching and negative for shrinkage. $\mathbf{b}$, Average yy (left)-, xy (middle)- and isotropic (right) components of stress around $\mathrm{a}+1 / 2$ defect obtained from experiments for NIH3T3 $(\mathrm{n}=$ 1,428 defects from 2 independent experiments).

Published online: 9 March 2021

https://doi.org/10.1038/s41563-021-00974-9

(c) The Author(s), under exclusive licence to Springer Nature Limited 2021

\section{Publisher Correction: Phonon renormalization in reconstructed $\mathrm{MoS}_{2}$ moiré superlattices}

Jiamin Quan, Lukas Linhart (D), Miao-Ling Lin, Daehun Lee, Jihang Zhu, Chun-Yuan Wang, Wei-Ting Hsu, Junho Choi (D), Jacob Embley, Carter Young (D), Takashi Taniguchi (D), Kenji Watanabe D, Chih-Kang Shih (D), Keji Lai, Allan H. MacDonald, Ping-Heng Tan (D), Florian Libisch (D) and Xiaoqin Li (D)

Correction to: Nature Materials https://doi.org/10.1038/s41563-021-00960-1, published online 22 March 2021.

In the version of this Article originally published, in Fig. $2 \mathrm{~b}$ the twist angle labels were incorrect for the top two spectra. The top spectrum was mistakenly labelled ' $2.0^{\circ}$ ' and the second-from-top spectrum was mistakenly labelled ' $1.0^{\circ}$ '; the labels should have been ' $20.0^{\circ}$ ' and ' $10.0^{\circ}$ ', respectively. These errors have now been corrected.

Published online: 6 April 2021

https://doi.org/10.1038/s41563-021-00998-1

(c) The Author(s), under exclusive licence to Springer Nature Limited 2021 